\title{
Multi-Objective Capacity Optimal Allocation of Hybrid Energy Storage System
}

\author{
Shize $\mathrm{Li}^{1,}$, , Suping $\mathrm{Gao}^{2,}$ band Chaojiang Guo, \\ ${ }^{1}$ Harbin Institute of Technology Shenzhen Graduate School, Shenzhen 518055, China; \\ ${ }^{2}$ School of Shenzhen Polytechnic, Shenzhen 518055, China \\ alsz532652692@126.com, bgaosup@szpt.edu.cn, c1062911600@qq.com
}

\begin{abstract}
Keywords: Hybrid Energy Storage; Optimized Allocation; Particle Swarm Optimization; Control Strategy
\end{abstract}

Abstract. For battery and super-capacitor hybrid energy storage system capacity configuration, the hybrid energy storage system multi-objective optimal allocation model is proposed. The optimization goals were economic cost, reliability of power supply,and the balance of supply and demand. The constraints were the battery's SOC(State of Charge), the super-capacity's terminal voltage and the converter's maximum power. Taking into account the influence of the energy control strategy, the two stage PSO(Particle Swarm Optimization) algorithm was used to configure the hybrid energy storage system capacity. Finally, the simulation results show that the proposed algorithm can converge quickly and reliably.

\section{Introduction}

With energy becoming increasingly tense and the needs of low-carbon energy development, The world are trying to develop new energy application mode. Energy storage technology as a key part of the new energy applications, and it's energy storage component selection and operation control strategies need to be addressed. Recently, lithium-ion batteries or lead-acid batteries are commonly used in distributed generation[1-2], but the relevant study found that the battery and super-capacitor hybrid configuration can effectively reduce the number of battery charge and discharge to extend battery life [3].

Energy storage capacity allocation is an important part of the planning and design stage of distributed generation system, and how to maintain the balance between supply and demand according to load demand and power generation determines the system's economy and reliability [4]. The optimal allocation scheme of hybrid energy storage for microgrids with multiple distributed power sources is analyzed in [5], but this optimal allocation only takes the energy cost as the objective function. The enhanced Pareto algorithm is applied in [6] to solve the multi-objective optimization problem of the hybrid microgrids including wind power, photovoltaic system, diesel generator, and battery storage. The optimal targets of the model are PV panels, wind turbines, diesel generators and battery, and the super-capacitors are not involved in the energy storage devices. An improved two-stage model algorithm is proposed in [7] to find the optimal solution in multiple scenarios. It is shown that the control strategy has some influence on the hybrid capacity allocation in the planning and design phase. The energy storage cost is reduced by considering the quasi-steady-state simulation of the control strategy in the whole design stage, but the power quality problem of the system is not taken into account. In the capacity optimal allocation model, most scholars use the HOMER software to design the system performance of different types of energy combination [8-9], which can be combined with the control strategy to simulate the operation of the whole life cycle. However, due to the limitations of HOMER software, only the capacity allocation of costs optimal can be obtained. The load characteristics of five typical microgrids in universities, industrial areas, residential areas, commercial areas and agricultural areas are analyzed in [10], and the capacity allocation model is designed from the point of user profitability and power supply reliability. However, both cases are single objective function, and the load shortage index is not taken into account. 
In summary, most of the scholars focus on the energy storage capacity configuration on a single device, while they do not have sufficient research on the hybrid energy storage system capacity allocation. Hybrid energy storage system involving energy and power type storage equipment requires a control strategies to control the imbalance power distribution. In order to make the optimal allocation results closer to the actual demand, this paper proposes a multi-objective energy storage capacity allocation model considering the life cycle cost, power supply reliability index, power shortage index, and the balance between supply and demand.

\section{Distributed Hybrid Energy Storage System}

Distributed power generation system uses active hybrid energy storage structure, and the super-capacitors and lead-acid batteries of the system through their respective converter connect to DC bus, as shown in Figure 1. Compared to the passive structure, active structure can be flexible control the charge and discharge power of super-capacitor and lead-acid battery. Photovoltaic system and wind power system through their respective maximum power converter connect to DC bus.

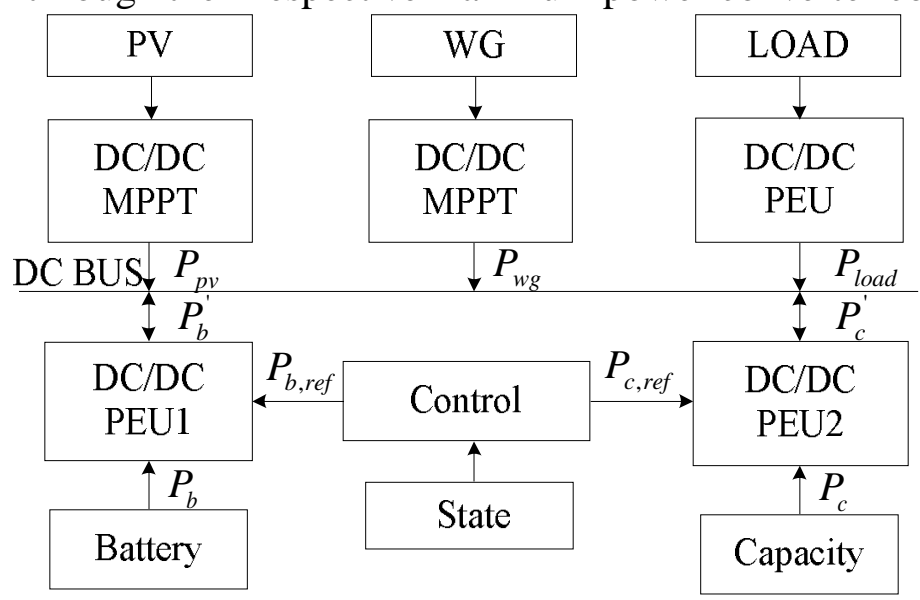

Fig.1 Isolated distributed generation system

In the independent hybrid distributed generation system with active hybrid energy storage, the energy from new energy sources needs to be dissipated in the system, that is, the system needs hold the dynamic equilibrium between supply and demand.

$$
\left\{\begin{array}{l}
P_{R E}-P_{H E S S}=P_{L O A D} \\
P_{R E}=P_{p v}+P_{w g} \\
P_{H E S S}=P_{b}^{\prime}+P_{c}^{\prime}
\end{array}\right.
$$

Where is the power of photovoltaic and wind turbine generation system, is the total power of the super-capacity and battery, is the power of load; Considering the efficiency of the converter, is the super-capacity power through the converter, is the battery power through the converter, is the power of photovoltaic system, is the power of wind turbine system.

\section{Capacity Optimization Configuration Model}

In the micro-network planning and design stage, the single-objective capacity optimization model tends to sacrifice other performance such as system reliability to reduce costs. By using multi-objective capacity optimization model, we can get a more reasonable configuration capacity [6], so this paper uses the multi-objective capacity optimization model. In the multi-objective optimization problem, a multi-objective optimization model is formed by linearly weighting multiple sub-objective functions into a single objective function, and then considering some constraints.

$$
\left\{\begin{array}{l}
\min f(x)=\lambda_{1} f_{1}(x)+\lambda_{2} f_{2}(x)+\lambda_{3} f_{3}(x) \\
x=\left[W_{b a t}, W_{u c}, P_{b a t}, P_{u c}\right]^{T} \\
\text { s.t. } \quad x \in X
\end{array}\right.
$$


Where $f(x)$ is the final objective function after aggregation, $f_{1}(x)$ is the cost sub-objective function, $f_{2}(x)$ is the power supply reliability and energy excess sub-objective function, $f_{3}(x)$ is supply and demand balance sub-objective function; $x$ is configuration Variable, including battery energy, super-capacitor energy, battery power and super-capacitor power; $W_{b a t}$ is battery energy, $W_{u c}$ is super-capacitor energy, $P_{b a t}$ is battery power, $P_{u c}$ is super-capacitor power; $X$ is constrains of variable $x$, including the SOC of the battery, the terminal voltage of super-capacity and the maximum power limitation; $\lambda_{1}, \lambda_{2}$ and $\lambda_{3}$ are the weights of sub-objective function respectively.

\section{Sub-Objective Function}

Cost objective function. Hybrid energy storage costs include initial construction costs and maintenance costs, taking the battery and super capacitor energy and power into account, the cost objective function is shown in equation (4).

$$
f_{1}(x)=c_{e 1} \frac{W_{u c}}{\eta_{1}}+c_{e 2} \frac{W_{b a t}}{\eta_{2}}+c_{m 1} P_{u c}+c_{m 2} P_{b a t}
$$

Where $W_{u c}$ and $W_{b a t}$ are the capacity of the super-capacity and battery respectively; $P_{u c}$ and $P_{b a t}$ are the power of the super-capacity and battery respectively; $\eta_{1}$ and $\eta_{2}$ are the efficiency of the super-capacity and the battery; $c_{e 1}$ and $c_{e 2}$ are the unit price of the super-capacity and the battery; $c_{m 1}$ and $c_{m 2}$ are the maintenance cost of the super-capacity and the battery.

Power supply reliability and energy excess objective function. If the microgrid is not connected to the grid, when the generating power is greater than the load power and the storage energy absorption, it will cause power waste. If the generating power and the stored energy discharge power is less than the load power, it will cause the system less reliable. Therefore, the power supply reliability and the excess energy objective function is expressed by sum of the difference of the battery power and the distributed power by the actual control strategy, as shown in equation (5).

$$
f_{2}(x)=\sum_{i=1}^{N}\left(\left(P_{N, b a t}-P_{b a t}(i)\right)^{2}+\left(P_{N, u c}-P_{u c}(i)\right)^{2}\right)
$$

Where $P_{b a t}(i)$ is the battery instantaneous power by control strategy allocates; $P_{u c}(i)$ is the super-capacity instantaneous power by control strategy allocates; $P_{N, b a t}$ is the rated power of the battery; $P_{N, u c}$ is the rated power of the super-capacity.

Supply-demand balance objective function. Hybrid energy storage systems need to ensure that the power changes of the battery and the super-capacitor to provide or absorb as small as possible to ensure system stability, so take the power variance of the battery and the super-capacitor as the goal.

$$
\left\{\begin{array}{c}
f_{3}(x)=\sum_{i=1}^{N}\left(P_{N, b a t}-P_{b a t}(i)-\Delta P_{a v g, b a t}\right)^{2} \\
+\sum_{i=1}^{N}\left(P_{N, u c}-P_{u c}(i)-\Delta P_{a v g, u c}\right)^{2} \\
\Delta P_{a v g, b a t}=\sqrt{\frac{\sum_{i=1}^{N}\left(P_{N, b a t}-P_{b a t}(i)\right)^{2}}{N}} \\
\Delta P_{a v g, u c}=\sqrt{\frac{\sum_{i=1}^{N}\left(P_{N, u c}-P_{u c}(i)\right)^{2}}{N}}
\end{array}\right.
$$


Where $P_{N, b a t}$ and $P_{N, u c}$ are the rated power of the battery and the super-capacity; $P_{b a t}(i)$ and $P_{u c}(i)$ are the battery and the super-capacity power by control strategy allocates; $\Delta P_{\text {avg bat }}$ and $\Delta P_{a v g, u c}$ are the power difference of the battery and the super-capacity.

\section{Sub-Objective Function Weight.}

The hybrid storage capacity configuration contains multiple sub-objective functions. Weigh the importance of each sub-objective function according to the actual situation to determine the weight of each sub-objective function by adaptive sort method. The difference between the different capacity configurations and the optimal configurations is shown in equation (7).

$$
\delta_{i}^{j}=f_{i}^{j}-f_{i}^{i} \quad i, j=1,2, \ldots, m
$$

Where $m$ is the number of objective function; $f_{i}^{j}=f_{i}\left(x_{j}\right)$ is the function value with different solution. The procedure of determining the weights of each sub-objective function as follows:

1) Suppose there are m sub-objective functions, the optimal value of the sub-objective function is solved according to the single objective optimal situation.

2) For the optimal solution of different objective function, solve the difference of this value and the other target value, as shown in equation (7). The optimal value of a sub-function in other functions is the second-best solution, and the difference between the value and the optimal solution is non-negative.

3) Using equation (8) to calculate the deviation of each sub-objective function.

$$
u_{i}=\frac{1}{m-1} \sum_{j=1}^{m} \delta_{i}^{j} \quad i=1,2, \ldots, m
$$

Because $\delta_{i}^{i}=0$, so the mean value was calculated according to $\mathrm{m}-1$.

4) Using equation (9) to calculate the weights of sub-objective function.

$$
\lambda_{i}=1-\frac{u_{i}}{\sum_{j=1}^{m} u_{j}} \quad i=1,2, \ldots, m
$$

5) As the deviation is non-negative, so the weights are always positive, and then normalize the weight as equation (10).

$$
\lambda_{i}=\frac{\lambda_{i}}{\sum_{j=1}^{n} \lambda_{j}} \quad i=1,2, \ldots, n
$$

Where $\mathrm{n}$ is the number of sub-objective function, $\lambda_{i}$ is weight of the sub-objective function, and $\sum_{i=1}^{m} \lambda_{i}=1$

\section{Capacity Allocation Constraints}

The constrains of the battery SOC. In order to prevent the reduce of the battery life and the super-capacitor life, limit the battery upper and lower power.

$$
S_{O C, \min } \leq S_{O C, i} \leq S_{O C, \max }
$$

Where $S_{O C, \min }$ is the lower SOC limit of the battery, generally about $20 \% \sim 30 \% ; S_{O C, \max }$ is the upper SOC limit of the battery, generally about $80 \% \sim 100 \%$ 。

The constrains of the super-capacity terminal voltage. In order to prevent the reduce of the super-capacitor life, make the upper and lower limits on the terminal voltage.

$$
V_{O C, \min } \leq V_{O C, i} \leq V_{O C, \max }
$$


Where $V_{O C, \text { min }}$ is the lower limit of the, generally about $10 \% \sim 20 \% ; V_{O C \text {, max }}$ is the upper voltage limit of the super-capacity, generally about $90 \% \sim 100 \%$.

The constrains of the maximum power. There is a minimum power requirement in the system to ensure that some critical loads can be uninterruptible power. At the same time the maximum power does not exceed the upper power limit of the converter.

$$
\left\{\begin{array}{l}
P_{N, u c}+P_{N, b a t} \geq P_{\min } \\
P_{N, u c} \leq P_{u c, \text { max }} \\
P_{N, b a t} \leq P_{b a t, \max }
\end{array}\right.
$$

Where $P_{N, b a t}$ and $P_{N, u c}$ are the rated power of the battery and the super-capacity; $P_{\min }$ is the minimum power demand; $P_{b a t, \max }$ and $P_{u c, \max }$ are the maximum power limit of the battery and the super-capacity.

\section{Capacity Allocation Optimization Algorithm}

This paper applied use two-stage optimization iterative strategy. In the first stage, the PSO algorithm calculate the three objective functions, and the difference between the value and the other objective functions. Then the weighting values of each particle are obtained by the deviation sorting method. In the second stage, each particle updates itself by tracking the two extremes, which are local optimal solution pBest and global optimal solution gBest. The above two phases are repeated until the termination condition is satisfied. The PSO algorithm take the updating algebra as the termination criterion.

For the problem of PSO easy to convergence early and poor global optimization ability, the following improvements are made[14]: At the beginning of the first stage, increase the damping perturbation for each particle position and velocity, so as to strengthen the global optimization ability and avoid premature convergence.

Fig. 2 shows that the process of solving the hybrid energy storage capacity optimization model by using the improved multi-objective particle swarm optimization algorithm. 


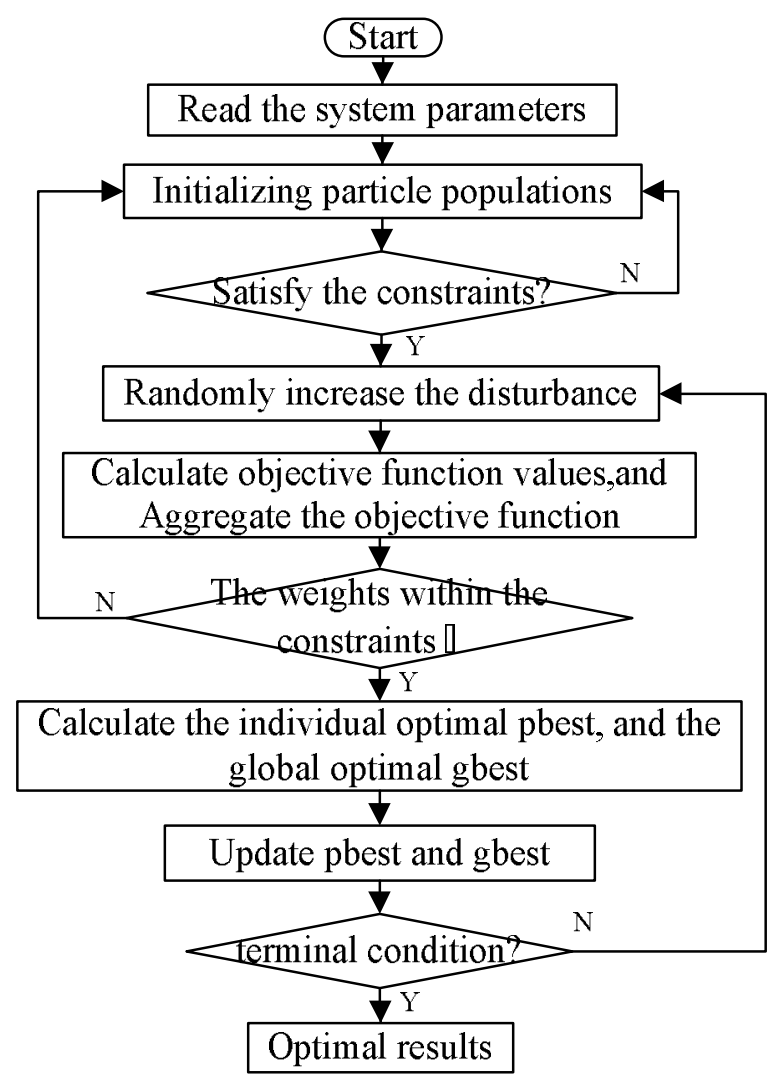

Fig.2 Optimization Model Solving Process

1)Firstly, initialize the capacity optimization configuration model. Reading the wind turbine, PV, hybrid energy storage device and PSO parameter, and randomly generate $\mathrm{N}$ group capacity configuration Solution Set.

$$
x_{i}=\left[W_{b a t, i}, W_{u c, i}, P_{b a t, i}, P_{u c, i}\right]^{T}
$$

2) Evaluation the capacity configuration of $\mathrm{N}$ groups. If it can not meet all constraints under full life cycle simulation, this configuration is invalid and should be reconfiguration;

3) Randomly increase the particle position and velocity damping perturbations. In order to overcome the problem of PSO is easy to fall into local optimum, increasing the damping disturbances.

4) Using sorting deviation calculate the weights and the function values.

5) Into the second stage. Update the historical optimal allocation and the total optimal configuration of $\mathrm{N}$ groups. After the $\mathrm{N}$ groups of configurations are evaluated, Select the optimal value by compare the $\mathrm{N}$ groups capacity configuration with the respective historical value. And then find the total particles' optimal value.

6) Using equation (15) to update the capacity configuration set .

$$
\left\{\begin{array}{l}
x_{i}(t)=x_{i}(t-1)+v_{i}(t-1), t=1,2, \ldots, K \\
v_{i}(t)=w x_{i}(t-1)+c_{1} r_{1}\left(x_{\text {pbest }}-x_{i}\right)+c_{2} r_{2}\left(x_{\text {gbest }}-x_{i}\right)
\end{array}\right.
$$

Where represents the number of iteration; is the configuration of batteries and super-capacitors after $t$ update; is the difference between the current solution and the last solution. is individual optimal solution; is total optimal solution; is inertia weight, and are acceleration factor, and is a random number between 0 and 1 .

7) Whether the last iteration number reaches $K$ value, which is the number of iterations after trade-off accuracy and computational complexity. If the iteration does not reach $\mathrm{K}$, go back to step 3 to continue; if the iteration reaches $\mathrm{K}$, the search stop and outputs the optimal solution of the battery and the super-capacitor.

$$
x_{o p}=\left[W_{b a t, o p}, W_{u c, o p}, P_{b a t, o p}, P_{u c, o p}\right]^{T}
$$


Where is the optimal solution after $\mathrm{k}$ iteration, is the optimal solution of the battery energy; is the optimal solution of the super-capacity energy; is the optimal solution of the battery power; is the optimal solution of the super-capacity power.

According to the hybrid energy storage control strategy, enumerate a series of filter time constants Circulating the above steps, then evaluate the Pareto border value by the total objective function value to find the optimal solution set.

\section{Case Study}

Select a demonstration micro-grid as a design case, and the system's energy storage equipment include batteries and super-capacitors. Tab.1 shows the data of annual photovoltaic, wind power, load power with one hour step by using HOMER software. One objective of stand-alone system has to satisfy the supply-demand balance, so it is necessary to calculate the supply-demand unbalance power. Fig.3 shows the typical power generation data, load data and supply-demand unbalance power.

Tab.1 Distributed Generation System Characteristic Data

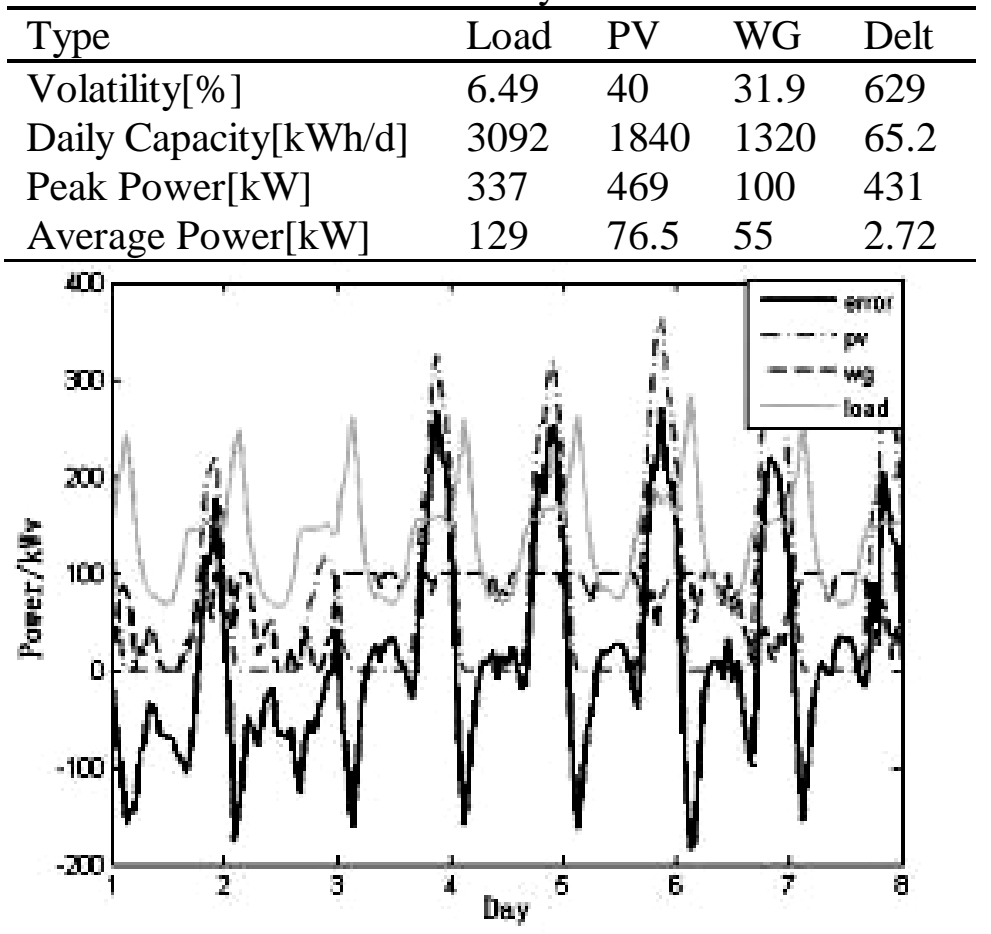

Fig.3 Typical Power Fluctuation Curve

Imbalance power break down into high and low parts after a low-pass filter, and they are stabilized by the super capacitors and batteries. Cut-off frequency choose $0.15 \mathrm{~h}, 0.25 \mathrm{~h}, 0.35 \mathrm{~h}$, and $0.45 \mathrm{~h}$. Fig. 4 shows the power fluctuations after the hybrid energy storage system stabilize the imbalance power. 


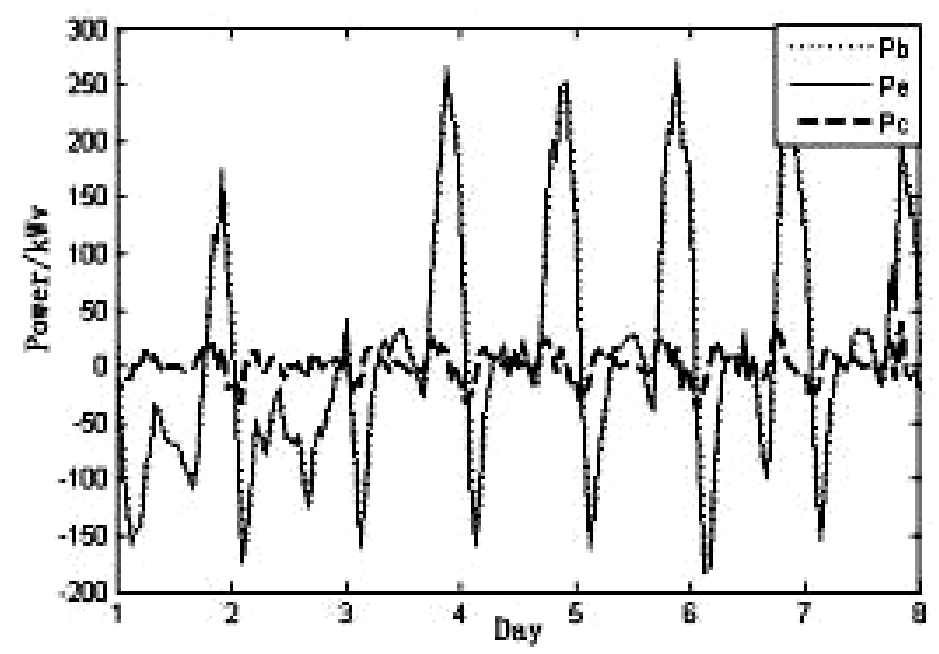

Fig.4 Super-capacitor and battery power fluctuation curve

Low-pass filter remove the the high-frequency fluctuations from the unbalanced power, and it can make the lead-acid battery's power becomes smooth. Therefore the lead-acid battery can avoid charge and discharge frequently. The super-capacitor can charge and discharge in ultra-high frequency, so it can stabilize the low peak and high frequency fluctuations.

Tab.2 shows that the multi-objective configuration model's constraints have the minimum required energy storage capacity, the power required in the optimal configuration, and the device parameter values are determined. Solving the optimal solution by using the optimal allocation algorithm proposed in this paper, and the PSO parameters are as follow, the population is 40; the maximum inertia weight is 0.9 ; minimum inertia weight is 0.4 ; learning factor is 2.0 ; maximum number of iterations is taken as 70 .

Tab.2 Mixed energy storage equipment parameter values

\begin{tabular}{lrr}
\hline Parameters & Battery & Super-capacity \\
\hline Unit Power Cost[yuan/kW] & 1000 & 40000 \\
Cycle Life Times & 1300 & 100000 \\
Charging Efficiency & 0.9 & 0.9 \\
Discharging Efficiency & 0.9 & 0.9 \\
Maximum SOC Limit & 0.8 & 0.9 \\
Minimum SOC Limit & 0.2 & 0.1 \\
\hline
\end{tabular}

The two stage PSO algorithm is used to get the optimal allocation result, as shown in Fig.5. Tab.3 represents the iterative convergence of particle swarm optimization with cost target as ordinate. From simulation, the algorithm can converge quickly at an average annual cost of 742,000 yuan. The battery's power and capacity are $60.8 \mathrm{~kW}$ and $110 \mathrm{kWh}$ respectively. The super-capacitor's power and capacity are $16.6 \mathrm{~kW}$ and $1 \mathrm{kWh}$ respectively. 


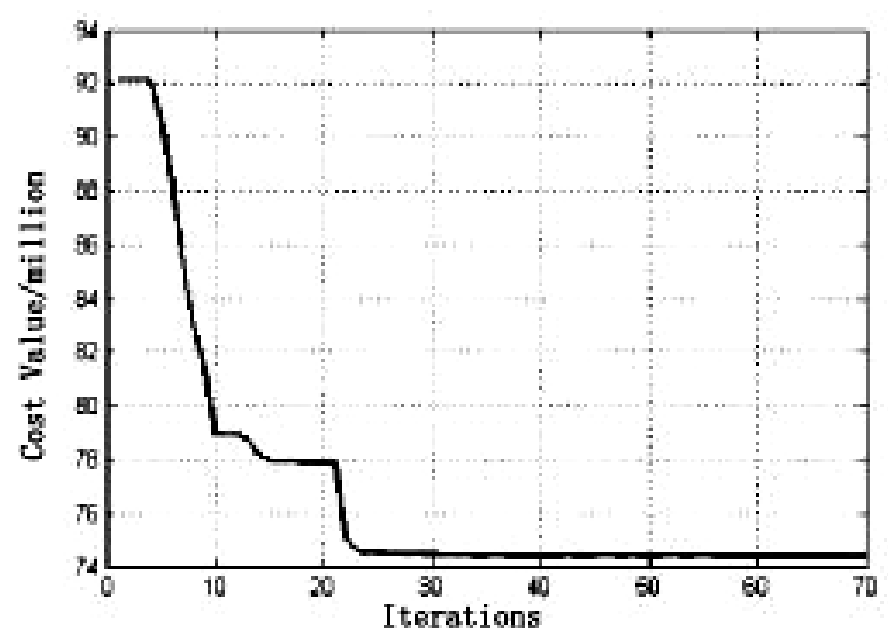

Fig. 5 Optimal Target Convergence Curve

Tab.3 Hybrid energy storage optimal configuration results

\begin{tabular}{lc}
\hline Index & Value \\
\hline Total Investment Cost[Million] & 74.2 \\
Excess Energy Rate[\%] & 0.0154 \\
Load Loss Probability[\%] & 0.6000 \\
Battery Rated Power[kW] & 60.8 \\
Battery Rated Capacity[kWh] & 110 \\
Super-Capacity rated Cower[kW] & 16.6 \\
Super-Capacity Rated Capacity[kWh] & 1 \\
\hline
\end{tabular}

\section{Conclusion}

This paper presents an optimization allcoation method to stabilizing power fluctuations in hybrid energy storage systems. The optimization method's optimization goals were the economic cost, reliability of power supply, and the balance of supply and demand. It's constraints were the battery's SOC, the super-capacity's terminal voltage and the converter's maximum power. The two stage PSO(Particle Swarm Optimization) algorithm was used to configure the hybrid energy storage system capacity.Finally, based on the data of power distributed generation data, the hybrid energy storage optimization configuration result is given by MATLAB simulation, and the simulation results show that the proposed algorithm has faster convergence.

\section{Acknowledgements}

This work was financially supported by Knowledge Innovation Program of Shenzhen Commission on Innovation and Technology(JCYJ20140718172055170)

\section{References}

[1] Haixue Lin. Applying Situation and Prospects of Modern Energy Storage Technology[J]. Journal of Power Supply, 2015, 13(5): 35-47(in Chinese).

[2] Kai Jiang, Haomiao Li, Wei Li, et al. Introduction of several kinds of energy storage batteries for grid[J]. Journal of Power System Automation, 2013, 37(1): 47-53(in Chinese).

[3] Shouping $\mathrm{Xu}$, Xiangjun $\mathrm{Li}$, dong Hui. Review on the development of large scale electrochemical energy storage system and its demonstration[J]. Journal of Electric Power Construction, 2013, 34(7):73-79(in Chinese). 
[4] Jun Xiao, Zequn Zhang, Pan Zhang, et al. A hybrid energy-storage capacity optimization method for optimizing the power of micro-network tie-lines[J]. Journal of Power System Automation, 2013, 38(12): 19-25(in Chinese).

[5] Kun Zhang, Chengqiong Mao, Junwen Xie. Optimized design of capacity allocation for composite energy storage system in wind farm[J]. Transactions of Chinese Society for Electrical Engineering, 2012, 32(25): 79-87(in Chinese).

[6] Ogunjuyigbe A S O, Ayodele T R, Akinola O A. Optimal allocation and sizing of PV/ Wind/ Split-diesel/ Battery hybrid energy system for minimizing life cycle cost, carbon emission and dump energy of remote residential building[J]. Applied Energy, 2016, 171(6): 153-171.

[7] Li Guo, Wenjian Liu, Bingqi Jiao, et al. Multi - objective optimization planning and design method for independent micro-network system[J]. Transactions of Chinese Society for Electrical Engineering, 2014, 34(4): 524-536(in Chinese).

[8] Hao Xiao, Wei Pei, Yanhong Yang, et al.Optimization of energy storage capacity of microgrid considering battery life and economical operation[J]. Journal of High Voltage Technology, 2015, 41(10): 3256-3265(in Chinese).

[9] Dufo-Lópeza R, Bernal-Agustína J L, Yusta-Lovo J M, et al. Multi-objective optimization minimizing cost and life cycle emissions of stand-alone PV-wind-diesel systems with batteries storage[J]. Applied Energy, 2011, 88(11): 4033-4041.

[10]Chong L W, Wong Y W, Rajkumar R K, et al. Hybrid energy storage systems and control strategies for stand-alone renewable energy power systems[J]. Renewable and Sustainable Energy Reviews, 2016(66): 174-189.

[11] Sainan Li. Capacity allocation of distributed generation and energy storage of microgrids considering grid constraints[D]. Beijing: Beijing Jiaotong University, 2016(in Chinese).

[12] Wei Wu, Shaojun Xie, Zhao Zhang, et al. Analysis and Design of Control Strategy for Combined-BDC Based Ultra-capacitors Energy Storage Systems[J]. Journal of Power Supply, 2016, 14(3): 83-93(in Chinese).

[13]Xingguo Tan, Hui Wang, Li Zhang, et al. Micro-grid energy storage complex multi-objective optimization and configuration methods evaluation [J]. Journal of Power System Automation, 2014, 38(8): 7-14(in Chinese).

[14]Qingyuan He, Chuanjiu Han. Improved particle swarm optimization algorithm with disturbance[J]. Computer Engineering and Applications, 2007, 43(7): 84-86(in Chinese).M.A. Green: High Efficiency Silicon Solar Cells (Trans Tech Publications, Switzerland 1987). 